\title{
Role of Bordetella bronchiseptica adenylate cyclase in nasal colonization and in development of local and systemic immune responses in piglets
}

\author{
Laurence HIBRAND-SAINT OYANT ${ }^{\mathrm{a}}$, Dorothée BOURGES ${ }^{\mathrm{a}}$, \\ Claire CHEVAlEYRE ${ }^{\mathrm{a}}$, Dominique RAZE ${ }^{\mathrm{b}}$, Camille LOCHT $^{\mathrm{b}}$, \\ Henri SALMON ${ }^{\text {a* }}$
}

\begin{abstract}
a Laboratoire Lymphocytes et Immunité des Muqueuses, Institut National de la Recherche Agronomique (INRA), Centre de Recherche de Tours, 37380 Nouzilly, France

b Laboratoire de Microbiologie Génétique et Moléculaire, INSERM U447, Institut Pasteur de Lille, 1 rue du Professeur Calmette, 59019 Lille, France
\end{abstract}

(Received 23 January 2004; accepted 2 August 2004)

\begin{abstract}
Two Bordetella bronchiseptica mutants, lacking the adenylate cyclase (Cya) or both Cya and pertactin (Prn), were compared with their parental strain NL1013 in their abilities to colonize the nose of neonate piglets and to induce local and systemic antibody responses against filamentous hemagglutinin (FHA) after intranasal (i.n.) inoculation. The number of bacteria recovered and the duration of infection in the nasal secretions were greater for the wild-type parent strain than for the Cya-deficient mutant, indicating that Cya plays an important role during B. bronchiseptica colonization of the nasal cavity. The double mutant did not colonize the nasal cavity and was less able to adhere to epithelial cells in vitro than the other two strains, supporting the hypothesis that Prn plays a major role in cell adhesion. In piglets inoculated with the wild type strain, anti-FHA IgM was found in the nasal secretions one week after inoculation, followed two weeks later by anti-FHA IgA; their presence was concomitant with decreases in bacterial counts. Anti-FHA IgG appeared at six weeks after infection in the serum. In contrast, i.n. inoculation with either mutant failed to induce a nasal secretory antibody response but did induce an earlier and higher IgM response in the serum than inoculation with the wild type strain. However, only the Cyadeficient mutant was able to prime the piglets for the development of a secondary nasal $\operatorname{IgM}$ and serum IgG response to FHA after intranasal inoculation with the wild type B. bronchiseptica.
\end{abstract}

Bordetella / piglets / FHA / immune response / IgA

\section{INTRODUCTION}

Bordetella bronchiseptica is a small aerobic, gram-negative bacterium, which colonizes the upper respiratory tract of many warm-blooded animal [11]. In addition to causing opportunistic infections in humans, it is a major pathogen among dogs and pigs. B. bronchiseptica infects newborn piglets during their first week of life, possibly due to the immaturity of their nasal mucosa $[5$, 16]. When co-infected with Pasteurella multocida, B. bronchiseptica-infected piglets develop atrophic rhinitis. The piglet

* Corresponding author: salmon@tours.inra.fr 
therefore constitutes a natural host model system with which to study B. bronchiseptica pathogenesis [1].

B. bronchiseptica synthesizes an array of virulence factors, which are similar but not identical to those of Bordetella pertussis, a strictly human pathogen responsible for whooping cough [26]. These virulence factors include adhesins (such as filamentous hemagglutinin (FHA), fimbriae and pertactin (Prn)), which may be involved in colonization and toxins (such as a bifunctional adenylate cyclase (Cya)-hemolysin, dermonecrotic toxin and tracheal cytotoxin), which may be involved in the formation of nasal and lung lesions. With the exception of tracheal cytotoxin, the production of these factors is regulated by the BvgAS two-component system $[3,41]$. This system modulates virulence in response to specific environmental signals [42].

Bordetella Cya is a member of the RTX family of bacterial exotoxins. It induces damage by catalyzing the production of supraphysiological amounts of cyclic-AMP. Infected mice clear Cya-deficient B. bronchiseptica strains from their lungs much faster than they do the cyclase-producing parent strains, suggesting that this toxin plays a major role in the initial phase of lung colonization in the mouse model [17]. In addition, naturally occurring isolates of B. bronchiseptica that do not produce the toxin are non-lethal upon intranasal (i.n.) infection in mice [12].

Most studies concerning the role of virulence factors in B. bronchiseptica colonization and the resulting immune response have been performed on mouse models. However, B. bronchiseptica colonizes the lower respiratory tract in mice, whereas in natural target species, such as piglets, $B$. bronchiseptica preferentially colonizes the nasal epithelium [1]. Following i.n. infection with B. bronchiseptica, mice develop anti-FHA antibodies in the serum and in the bronchoalveolar lavage fluid $[12,13]$. B. bronchiseptica FHA has a molecular mass and hemagglutination properties that are similar to those of B. pertussis FHA and the two strains share the same epitopes $[18,27,28]$. As in mice, anti- $B$. bronchiseptica antibodies are also frequently found in the sera of porcine populations.

We studied the role of the B. bronchiseptica virulence factors, Cya and Prn, in nasal colonization and the impact of these factors on the immune responses in neonate piglets. The ability of two strains, lacking either Cya or Cya and Prn, to colonize the upper respiratory tract and to induce local and systemic anti-FHA immune responses was compared to that of the wild-type parent strain.

\section{MATERIALS AND METHODS}

\subsection{Bacterial strains and growth conditions}

The bacterial strains and plasmids used in this study are listed in Table I. The Bordetella strains were grown at $37^{\circ} \mathrm{C}$ on plates with a Bordet-Gengou agar base (Difco, Cergy-Pontoise, France) supplemented with $1 \%$ glycerol and $10 \%$ sheep blood. Liquid cultures were grown in Stainer-Scholte medium [39]. There was no difference in the growth curves between mutants.

When necessary, antibiotics were included in the growth media at the following concentrations (in micrograms per milliliter): kanamycin, 25; gentamicin, 15; chloramphenicol, 30; streptomycin, 100; tetracycline, 20. The Escherichia coli strains were grown at $37{ }^{\circ} \mathrm{C}$ in Luria-Bertani medium [29] or on solid media obtained by the addition of Bacto-Agar (1.5\% wt/vol; Difco, Cergy-Pontoise, France) to the culture.

\subsection{Isolation of $B$. bronchiseptica mutant strains}

Plasmid DNA was routinely isolated by the alkaline lysis method [36]. Restriction endonucleases were obtained from Roche (Meylan, France) or New England Biolabs (Ozyme, Saint-Quentin-en-Yvelines, France) 
Table I. Bacterial strains and plasmids.

\begin{tabular}{|c|c|c|}
\hline Strain or plasmid & Relevant features $^{a}$ & Source or reference \\
\hline \multicolumn{3}{|l|}{ Strains } \\
\hline E. coli XL1-Blue & High efficiency transformation; $\mathrm{Tc}^{\mathrm{r}}$ & Stratagene \\
\hline E. coli $\mathrm{DH} 5 \alpha$ & High efficiency transformation & Invitrogen \\
\hline B. pertussis BPSM & Derivative of Tohama I rps $L ; \mathrm{Sm}^{\mathrm{r}} \mathrm{Nal}^{\mathrm{r}}$ & {$[27]$} \\
\hline \multicolumn{3}{|l|}{ B. bronchiseptica } \\
\hline NL1013 & & {$[2]$} \\
\hline NL1013 hem $^{-}$ & Spontaneous variant of NL1013 & This study \\
\hline NL1013 cyaB int & NL1013-derivative, disrupted in the $c y a B$ gene 1 & This study \\
\hline \multicolumn{3}{|l|}{ Plasmids } \\
\hline pZERO2 & High copy number vector; $\mathrm{Km}^{\mathrm{r}}$ & Invitrogen \\
\hline pJQ200mp18 & Bordetella suicide vector, contains $E$. coli rpsL; $\mathrm{Gn}^{\mathrm{r}}$ & [34] \\
\hline pBBR1MCS & Broad-host-range vector; $\mathrm{Cm}^{\mathrm{r}}$ & {$[22]$} \\
\hline pBBPG & $\begin{array}{l}\text { pBBR1MCS bearing a } X h o \mathrm{I}-B s p \mathrm{HI} \text { fragment of the } \\
\text { B. pertussis BPSM porin gene promoter region (Ppor) [4] } \\
\text { fused to the } g f p \text { gene [10] on a BspHI-NsiI fragment. }\end{array}$ & This study \\
\hline pCYA1 & pJQ200mp18-derivative bearing a 924-bp 5' region of cyaA & This study \\
\hline pCYA2 & PZERO2-derivative bearing a 972-bp 3' region of $c y a B$ & This study \\
\hline pCYA3 & $\begin{array}{l}\text { pCYA1-derivative with a } 1068 \text {-bp fragment insertion } \\
\text { containing the } 3 \text { ' region of } c y a \mathrm{~A}\end{array}$ & This study \\
\hline pCYA4 & pCYA3-derivative with the Ppor- $g f p$ insertion & This study \\
\hline
\end{tabular}

${ }^{a} \mathrm{Tc}^{\mathrm{r}}, \mathrm{Sm}^{\mathrm{r}}, \mathrm{Nal}^{\mathrm{r}}, \mathrm{Km}^{\mathrm{r}}, \mathrm{Gn}^{\mathrm{r}}$ and $\mathrm{Cm}^{\mathrm{r}}$ : resistance to tetracycline, streptomycin, nalidixic acid, kanamycin, gentamicin and chloramphenicol, respectively.

and used according to the standard procedures [36]. DNA fragments were sequenced using an ABI PRISM Dye Terminator Cycle Sequencing kit and an ABI PRISM 377 sequencer (PE Applied Biosystems, Warrington, United Kingdom) with a combination of universal, reverse and customsynthesized primers. Polymerase chain reactions (PCR) were carried out using Vent $_{R}$ DNA polymerase (New England Biolabs, Inc., Beverly, MA, USA). All cloning steps were done using E. coli XL1-Blue.

Oligonucleotides CyaCU54 (5'-ATATGAGCTCTTCGGCATGGTAGCGGTCCAG-3') and L928 (5'-ATATTCTAGATGTGTAGCGCTCAGAACCTCA-3') were used to amplify a fragment located $974 \mathrm{bp}$ upstream of the ATG translational start codon of the cyaA gene from B. bronchiseptica NL1013. The resulting fragment was digested with $S a c I$ and $X b a \mathrm{I}$ and inserted into pJQ200mp18 (digested by the same restriction endonucleases) to generate pCYA1. Oligonucleotides U6233 (5'-ATTATCAAGACTGGTCATGCTGGCTCGCTAT-3') and L7205 (5'-ATTAGTCG ACGCTGACGGTCTCCACCAGAAA-3') were used to amplify a 972-bp fragment located 132 bp downstream of the translational stop codon of the cyaA gene of NL1013, which corresponds to the 5' portion of the cyaB gene. The resulting blunt-ended fragment was inserted into pZERO2 (digested with EcoRV) to generate pCYA2. pCYA2 was digested with $\mathrm{BamHI}$ and $\mathrm{XbaI}$ to isolate a fragment bearing the downstream region of cyaA. pCYA3 was obtained by inserting this fragment into pCYA1. pCYA3 was then digested with $\mathrm{XhoI}$ and NsiI, and the Ppor- $g f p$ fragment (isolated from $p B B P G$ digested with $\mathrm{XhoI}$ and NsiI) was inserted 
to generate pCYA4.pBBPG is a pBBR1MCSderivative bearing the $B$. pertussis BPSM porin gene promoter region (Ppor) [25] fused to the $g f p$ gene [10]. To construct pBBPG, a 217-bp DNA fragment spanning Ppor was amplified using $B$. pertussis chromosomal DNA as the template and the oligonucleotide primers 5'-TATA CTCGAGCCCGCGCGCGATTCCGGATT-3' and 5'-TTTTTCATGAAAGAAATCTCCGT TGATTTG-3', which contain the XhoI and the $B s p H I$ sites (underlined), respectively. The $g f p$ gene was amplified from pEVET 10 using oligonucleotides 5'-CTGCTCATGAGTAAAGGAGAAGAACTTTTC-3' and 5'-TATAATGCATCCCGGGGATCCTCTAGAGTCGACCT-3', which contain the $B s p H I$ and $N s i$ sites (underlined), respectively. pEVET10 is a pUC18-derivative containing the PstI fragment, which includes the $g f p$ gene, from pGFM10.1 [23]. The amplified fragments were then digested with $X h o \mathrm{I}-B s p \mathrm{HI}$ and $B s p \mathrm{HI}-N s i \mathrm{I}$, respectively, and inserted into $\mathrm{pBBR} 1 \mathrm{MCS}$ (digested with $\mathrm{XhoI}$ and NsiI), to yield pBBPG.

The B. bronchiseptica NL1013 wild type strain was then transformed with the pCYA4 suicide vector. The recombinant strain, called B. bronchiseptica NL1013 cyaB int, which had lost the hemolytic phenotype, was analyzed by Southern-blotting to confirm that $c y a B$ had been disrupted by the integration of pCYA4. The Southern blots were carried out using the 465-bp PstI fragment of pCYA3 as a probe. Immunoblotting with anti-FHA and anti-Cya revealed that this recombinant strain secreted FHA at levels similar to those of the parent strain, but secretion of Cya was impaired. The integrated plasmid encodes a functional copy of the $g f p$ gene, since the recombinant strain was fluorescent on BG plates under long wave UV (365 nm) irradiation.

In addition to $B$. bronchiseptica NL1013 $c y a B$ int, we also isolated a spontaneous non-hemolytic kanamycin-resistant variant, named B. bronchiseptica NL1013 hem . This non-hemolytic variant still produced
FHA and had a colony morphology similar to that of the parent strain, indicating that the BvgAS system was fully functional in this variant. However, immunoblot analyses using anti-Prn antibodies indicated that this strain produces only trace amounts of Prn.

\subsection{Bacterial cell adhesion assays}

HeLa cells $\left(2.5 \times 10^{5}\right.$ cells per $\left.\mathrm{mL}\right)$, a commonly used epithelial cell line [37, 40], were seeded in Lab-Tek chamber slides (Nunc, Roskilde, Denmark) and incubated under $5 \% \mathrm{CO}_{2}$ at $37{ }^{\circ} \mathrm{C}$ in Dulbecco modified Eagle medium supplemented with 5\% fetal calf serum (Gibco, Cergy-Pontoise, France), $2 \mathrm{mM}$ glutamine, $100 \mathrm{IU} / \mathrm{mL}$ penicillin and $100 \mu \mathrm{g} / \mathrm{mL}$ streptomycin. After one day, the culture medium was removed, and the cells were fixed with $1 \%$ glutaraldehyde in PBS for $30 \mathrm{~min}$ at $4{ }^{\circ} \mathrm{C}$. The LabTek chamber slides were then washed with PBS followed by methanol. To saturate the non-specific sites, the slides were incubated for $1 \mathrm{~h}$ at $37^{\circ} \mathrm{C}$ with $0.2 \mathrm{~mL}$ PBS containing $1 \%$ BSA. After two washes, $0.2 \mathrm{~mL}$ of bacterial cell suspension (at $2.5 \times 10^{7}$ cells $/ \mathrm{mL}$ and in the exponential phase of growth) was added to a Lab-Tek chamber containing $10^{5} \mathrm{HeLa}$ cells. After incubation for $30 \mathrm{~min}$ at $37^{\circ} \mathrm{C}$ and five washings to remove nonadherent bacteria, the cells were fixed as described above and stained for $1 \mathrm{~h}$ with $1 \%$ (vol/vol) Giemsa (Rhône-Poulenc, Villiers St Paul, France). The cells were then examined with a light microscope (magnification $100 \times)$. The numbers of adherent bacteria per cell were determined for at least 25 cells. E. coli DH5 $\alpha$ was used as a negative control and did not adhere under these conditions.

\subsection{Animals and housing}

The initial inoculations were carried out on inbred miniature histocompatible $\mathrm{d} / \mathrm{d}$ piglets (between five and nine days old), bred in Nouzilly (France) [20]. Before the start of the experiment, the herd was free from $B$. bronchiseptica and of all clinical 
signs of atrophic rhinitis. Nevertheless, some sows, as well as their progeny had anti-FHA IgM and IgG in their serum. The three inoculated litters ( $n=3$ piglets for the litter infected with the NL1013 wild type and cyaB int strains, $n=4$ for the hem ${ }^{-}$strain) and their mothers were kept in separate chambers in an isolated piggery. Noninfected piglets from another litter $(n=2)$ were kept in a different isolated chamber and were used as negative control to assess the level of any contamination.

\subsection{Intranasal inoculation of piglets}

The piglets were i.n. inoculated by inserting the tip of a $1 \mathrm{~mL}$ syringe and injecting $0.1 \mathrm{~mL}$ of bacterial suspension into each nostril. The piglets were held so that their snouts were tilted backwards for $30 \mathrm{~s}$. The suspensions injected into each nostril contained $10^{7}$ CFU of B. bronchiseptica NL1013 wild type or $10^{8} \mathrm{CFU}$ of one of the mutant strains. For the wild type strain and negative control, $0.1 \mathrm{~mL}$ of bacteria and PBS, respectively, were injected, as described above, up each nostril of three 5-day-old piglets (comprising one litter).

Piglets initially inoculated with B. bronchiseptica NL1013 hem ${ }^{-}(n=3)$ or NL1013 cyaB int $(n=3)$ at one week of age, were then infected at 10 weeks of age with B. bronchiseptica NL1013. Unprimed piglets of the same age $(n=2)$ served as controls: no bacteria were re-isolated from the nasal cavities of any of these pigs.

\subsection{Collection and treatment of samples}

Samples of nasal secretions were taken from each nostril at the time points indicated in Figure 1 using a cotton swab of the same weight. The mucus was extracted from the swab with $1 \mathrm{~mL}$ of PBS containing $0.05 \%$ Tween-20. The extract was centrifuged at $6000 \times g$ for $15 \mathrm{~min}$, and the supernatants were stored at $-20{ }^{\circ} \mathrm{C}$ and used for

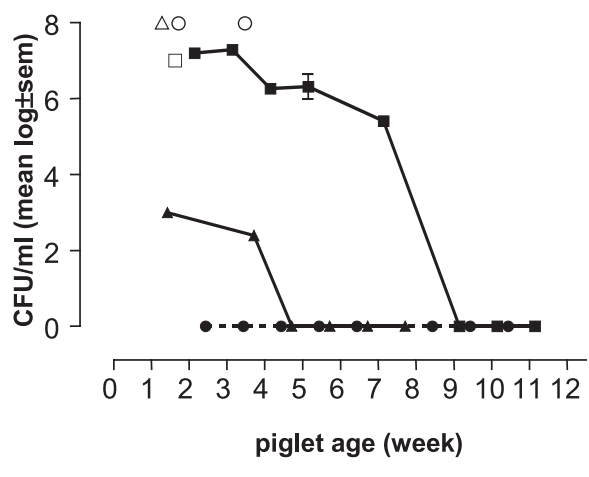

Figure 1. Colonization kinetics of B. bronchiseptica in the nasal cavity of piglets. Piglets from three separate litters were i.n. inoculated at the indicated time (open symbol) as follows: the first litter ( $n=3$ piglets) was inoculated with the $B$. bronchiseptica wild type $(\boldsymbol{\square})$, the second litter ( $n=3$ piglets) was inoculated with the NL1013 cyaB int $(\mathbf{\Delta})$ and the third litter $(n=4$ piglets $)$ was inoculated with the NL1013 $\operatorname{hem}^{-}(\mathbf{Q}, n=$ 4 piglets). At the time points indicated, samples of the nasal secretions were collected from both nostrils using cotton swabs and then plated onto Bordet-Gengou agar. The number of colonies on these plates were counted and an average of the values from these two nostrils was used so that a single averaged value was obtained for each piglet, thus $n=3$ for the NL1013 wild type, $n=$ 3 for NL1013 cyaB int strain and $n=4$ for the hem $^{-}$strain. The results are expressed as the mean $\log \mathrm{CFU} \pm$ sem per $\mathrm{mL}$ of nasal extracts. A fourth litter (two piglets) was i.n. inoculated with PBS and no bacteria were re-isolated from the nasal cavities of any of these pigs (data not shown).

the antibody measurements. The pellets were used to determine viable bacterial counts.

The bacteria recovered in the pellets were resuspended in PBS and plated onto BG agar supplemented with $10 \mu \mathrm{g} / \mathrm{mL}$ clindamycin and $25 \mu \mathrm{g} / \mathrm{mL}$ fungizone. After $48 \mathrm{~h}$ at $37^{\circ} \mathrm{C}$, the $\mathrm{B}$. bronchiseptica colonies were counted. For each piglet, the results are expressed as the average of the values (number of bacteria or antibodies level) from both nostrils.

The sera were separated from blood collected from the jugular vein and stored at $-20{ }^{\circ} \mathrm{C}$ until antibody determination. 
The samples of the nasal secretions and sera were collected from all of the piglets studied prior to inoculation to evaluate the preexisting levels of anti-FHA antibodies.

\subsection{Enzyme-linked immunosorbant assays}

FHA was purified from the culture supernatants by Heparin-Sepharose chromatography as described previously [28]. Maxisorb 96-well plates (A/S, Nunc, Roskilde, Denmark) were incubated with $100 \mu \mathrm{L}$ of $5 \mu \mathrm{g} / \mathrm{mL}$ FHA in carbonate buffer $(0.05 \mathrm{M}$, $\mathrm{pH}$ 9.6) for $2 \mathrm{~h}$ at $37^{\circ} \mathrm{C}$, after which they were washed four times with PBS containing $0.05 \%$ Tween-20 (PBS-T). A blocking step was then carried out by incubating the plates with PBS-T containing 5\% BSA for $1 \mathrm{~h}$ at $37{ }^{\circ} \mathrm{C}$. After four further washes, diluted nasal secretion or serum samples were added to the plates in duplicate and the plates were incubated overnight at $4{ }^{\circ} \mathrm{C}$. One-hundred microliters of peroxidase-conjugated rabbit anti-swine IgG (1/15000) (Sigma, A 7042, Saint-Louis, USA), mouse monoclonal anti-swine $\alpha$ chain $(1 / 8000)$ and goat anti-swine $\mu$ chain (1/250) (Kirkegaard-Perry laboratories, Gaithersburg, USA) were added to each well. After washing, $100 \mu \mathrm{L}$ of a substrate solution containing $40 \mathrm{mM}$ [2.2'-azino-di-(3-ethylbenzthiazoline-sulfonate)] diammonium salt (Boehringer Mannheim, Germany) and $3 \% \mathrm{H}_{2} \mathrm{O}_{2}$ in citrate buffer (100 mM, pH 4.2) was added. After $1 \mathrm{~h}$ at room temperature, optical density was read at $415 \mathrm{~nm}$ with Labsystems Multiscan RC (Labsystems, Cergy-Pontoise, France).

Sigmoidal standard curves for anti-FHA IgA, IgG and IgM were obtained from the titration of a pooled serum and a pooled nasal secretion sample obtained from two piglets eight weeks after infection with B. bronchiseptica NL1013. The absorbance values were adjusted by linear regression to form parallel curves after log transformation of the dilutions.
The concentrations of the anti-FHA IgM, IgG and $\operatorname{IgA}$ in the samples were determined by reference to the appropriate standard curve for each isotype. The concentrations are defined as ELISA titers, which correspond to the dilution that resulted in an absorbance equal to two standard deviations above the mean background obtained with non-immune serum or nasal secretions.

\subsection{Statistical analyses}

The differences in the proportions of cell bound bacteria between strains were assessed by the $\chi^{2}$ test and differences in the means of bacteria were assessed by variance analysis and Bonferroni tests. ELISA titers were compared by the Student $t$ test for paired samples, and multigroup comparisons of different time points were evaluated by variance analysis and the significant differences between the means were determined using the Bonferroni test. All calculations and curves were done using GraphPad Prism 2.01 software (GraphPad software, Inc., San Diego, CA, USA).

\section{RESULTS}

\subsection{Nasal colonization by $B$. bronchiseptica strains}

To assess the colonization of the bacteria in the upper respiratory tract, the amount of bacteria present in the nasal secretions was measured over the 10 week-period following i.n. inoculation (Fig. 1). During the first two weeks after i.n. inoculation, the parental wild-type strain remained at a level of $10^{7} \mathrm{CFU} / \mathrm{mL}$ and then decreased slowly during the following four weeks. The numbers then rapidly dropped during the following two weeks, to reach undetectable levels eight weeks after inoculation. During this period, we did not observe any clinical signs of respiratory disease nor atrophic rhinitis. The numbers of the Cya-deficient mutant B. bronchiseptica NL1013 cyaB int decreased much faster during the first week 
Table II. B. bronchiseptica adherence to HeLa cells ${ }^{\mathrm{a}}$.

\begin{tabular}{lcc}
\hline \multirow{2}{*}{ B. bronchiseptica strain } & \multicolumn{2}{c}{ HeLa cells with adherent bacteria } \\
\cline { 2 - 3 } & Cell (\%) & Numbers $^{\mathrm{b}}$ of bacteria/cell (mean \pm sem) \\
\hline NL1013 & 100 & $7.4 \pm 0.9$ \\
NL1013 cyaB int $_{\text {NL1013 } \text { em }^{-}}$ & 74 & $4 \pm 0.5^{* *}$ \\
\hline
\end{tabular}

a The results are representive of two independant experiments.

* Difference between the proportion of HeLa cells binding B. bronchiseptica NL1013 hem ${ }^{-}$and NL1013, $p<0.05$ by $\chi^{2}$ test.

${ }^{b}$ Each value represents the mean \pm sem of the number of bacteria per cell, counts were conducted on at least 25 cells for each strain.

** Differences between mutant strains and NL1013, $p<0.01$ by the Bonferroni test.

than those of the wild type, from $10^{8}$ to $10^{2}$. The number of bacteria then remained constant for two weeks and the nasal cavity was cleared of this strain between 3 and 4 weeks after inoculation. The double mutant $B$. bronchiseptica NL1013 hem $^{-}$, deficient for both Cya and Prn, was not recovered at any time point from the nasal cavity, even after a second i.n. inoculation.

\subsection{In vitro adherence of $B$. bronchiseptica strains}

To test whether the differences observed in the in vivo colonization and the persistence between the different strains result from differences in adhesion to epithelial cells, adherence assays were performed on HeLa cells. The results summarized in Table II show that the number of adherent bacteria was at least two times lower for the double mutant and single mutant than for the wild type $(p<0.01)$. In addition, the difference in the proportion of cell binding bacteria was much lower for the double mutant than for the single mutant $(p<0.05)$.

\subsection{Kinetics of local immune responses after inoculation with $B$. bronchiseptica strains}

The i.n. inoculation of one week-old piglets with B. bronchiseptica NL1013 induced a strong local immune response (Fig. 2a), characterized by production of anti-FHA IgM within one week of inoculation, fol- lowed two weeks later by a rise in anti-FHA IgA $(p<0.001)$. There was no significant rise in anti-FHA IgG levels $(p<0.001)$. There were three peaks in anti-FHA IgM levels at 2,5 and 8 weeks after infection. Similarly, three peaks in anti-FHA IgA were observed, each delayed by two weeks as compared to the anti-FHA IgM peaks.

The first anti-FHA IgM peak corresponds to the initial (slow) decrease in bacterial counts in the nasal secretions, whereas the first anti-FHA IgA peak corresponds to the rapid decrease in bacterial counts. The third peaks in both IgM and IgA correspond to the total clearance of the bacteria (Figs. 1 and $2 \mathrm{a}$ ).

After i.n. inoculation of either B. bronchiseptica NL1013 cyaB int or hem ${ }^{-}$, no nasal anti-FHA antibody was detected, regardless of the presence of bacteria in the nasal secretions (Figs. 2b and 2c).

\subsection{Systemic immune responses after i.n. inoculation with $B$. bronchiseptica strains}

The systemic immune response to i.n. inoculation with the $B$. bronchiseptica NL1013 wild type differed significantly from the local immune response. The first rise in anti-FHA antibodies occurred in IgG at 6 weeks of age (5 weeks after inoculation). There was no rise in the sera levels of anti-FHA IgM or IgA until 10 weeks after inoculation (Fig. 3a). 
(a) wt

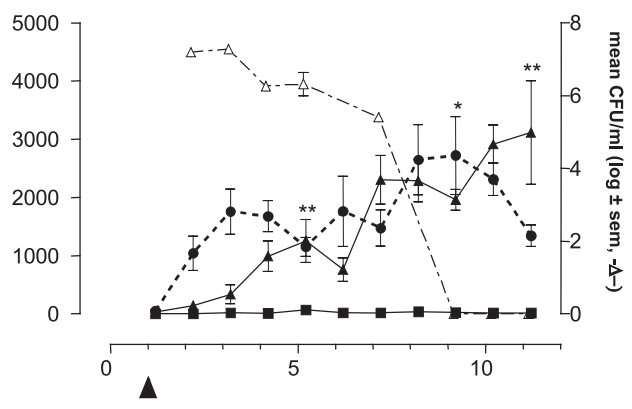

(b) cyaB int

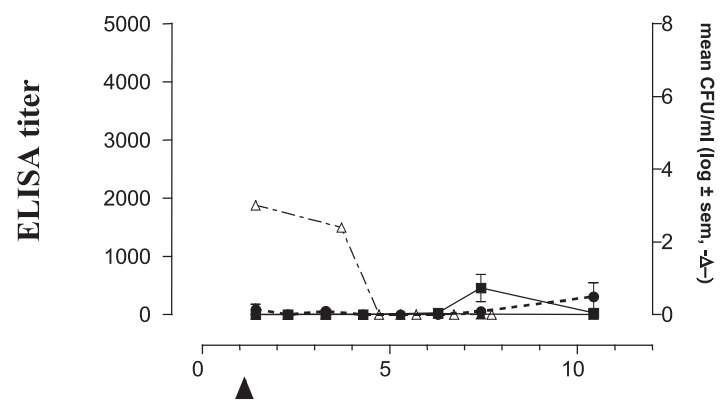

(c) hem-

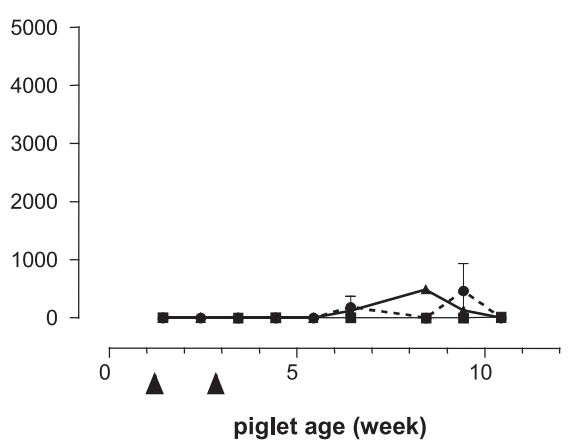

Figure 2. Kinetics of the anti-FHA antibody response in the nasal secretions. Anti-FHA $\operatorname{IgA}(\boldsymbol{\Delta})$, $\operatorname{IgG}(\boldsymbol{\square})$ and $\operatorname{IgM}(\bullet)$ levels were measured at the time points indicated in nasal secretions. The results are expressed as the mean titer \pm sem for each isotype. The same piglets as in legend Figure 1: (a) Piglets inoculated with $B$. bronchiseptica NL1013 wild type $(n=3)$, (b) Piglets inoculated with NL1013 cyaB int $(n=3)$, (c) Piglets inoculated with NL1013 hem he $^{-}(n=4)$. The titers obtained for each strain per nostril were averaged (thus: $n=3$ for the NL1013 wild type and $c y a B$ int strains and $n=8$ for the NL1013 hem $^{-}$strain). The arrowheads indicate the time of bacterial inoculation. The first sample was taken before inoculation to evaluate the pre-existing levels of anti-FHA antibodies. A fourth litter (two piglets) was i.n. inoculated with PBS and no antibody level was detected in any of these pigs (data not shown). Data points marked with * or $* *$ represent antibody levels that are significantly different from those obtained before inoculation at $p<0.005$, $*$ or $p<0.001$, ** using variance analysis and Bonferroni test for mean comparisons. 
(a)

wt

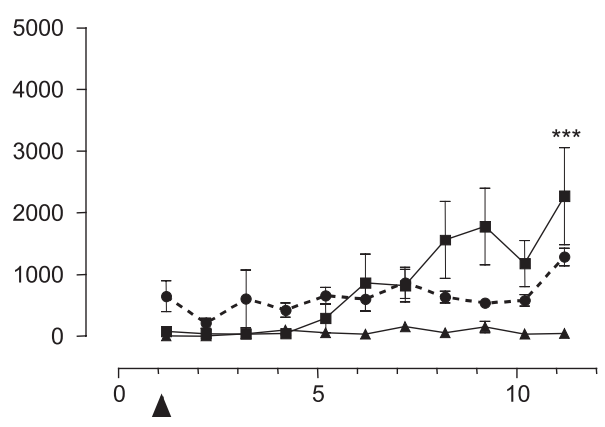

(b)

cya $B$ int

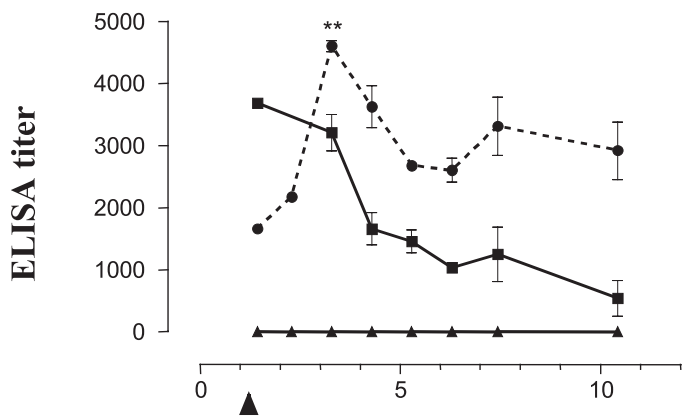

(c)

hem $^{-}$

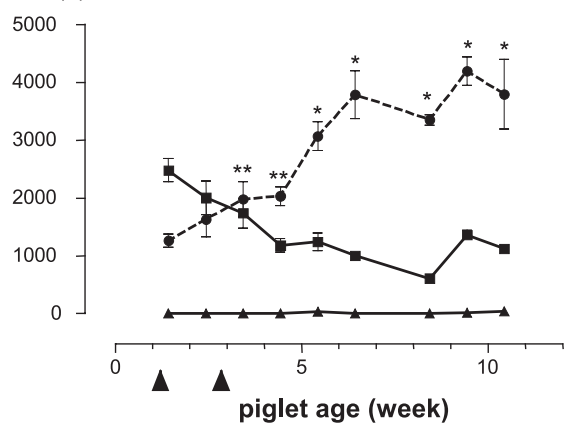

Figure 3. Kinetics of the anti-FHA antibody response in the sera. Anti-FHA $\operatorname{IgA}(\boldsymbol{\Delta}), \operatorname{IgG}(\boldsymbol{\square})$ and $\operatorname{IgM}(\bullet)$ levels were measured in the sera at the time points indicated. The results are expressed as the mean titer \pm sem for each antibody in (a) piglets inoculated with B. bronchiseptica NL1013 wild type $(n=3)$, (b) piglets inoculated with NL1013 cyaB int $(n=3)$, (c) piglets inoculated with NL1013 hem $^{-}(n=4)$. The triangle indicates the time of bacterial inoculation. Data points marked with *, $* *$ or $* * *$ represent antibody levels that are significantly different from those obtained before inoculation at $* p<0.001, * * p<0.01$ and $* * * p<0.05$ using the Bonferroni test. The first sample was taken before inoculation to evaluate the pre-existing levels of anti-FHA antibodies. A fourth litter (two piglets) was i.n. inoculated with PBS and no increase of antibody level was detected in any of these pigs (data not shown). 
After i.n. inoculation with NL1013 cyaB int, the level of anti-FHA IgM increased significantly and peaked two weeks after inoculation (Fig. 3b). The levels of this antibody then decreased during the following two weeks and then remained at a constant level until 10 weeks after infection. No antiFHA IgA was detected in the serum during the study period and there was no rise in IgG levels.

After i.n. inoculation with the double mutant NL1013 $\mathrm{hem}^{-}$, the anti-FHA IgM increased to a level which was higher than that of the preexisting maternally derived antibodies. No anti-FHA IgA was detected in any of the animals and the level of maternally derived anti-FHA IgG decreased steadily over the 10 -week study period.

\subsection{Nasal and serum antibody responses after a second infection with $B$. bronchiseptica NL1013}

Neither B. bronchiseptica mutant resulted in anti-FHA antibody production in the nasal secretions. We investigated whether this was due to the induction of local tolerance or to the absence of local priming by inoculating the piglets given the $B$. bronchiseptica NL1013 hem $^{-}$or NL1013 cyaB int inoculum at one week of age with $B$. bronchiseptica NL1013 at 10 weeks of age. As a control, unprimed piglets of the same age were also inoculated with the wild type. No bacteria were isolated from the nasal secretions of any of these piglets.

Initial inoculation with NL1013 cyaB int primed the piglets to mount an anti-FHA IgM response in the nasal secretions and an $\mathrm{IgG}$ response in the serum. This $\mathrm{IgG}$ serum response appeared to occur at the expense of IgM since the level of IgM in the serum decreased concomitantly with the increase of $\mathrm{IgG}$, suggesting a switch from IgM to IgG (Figs. 4a and 4d).

In piglets initially inoculated with the double mutant NL1013 $\mathrm{hem}^{-}$, exposure to the wild type strain, did not induce a detect- able anti-FHA response (Figs. 4b and 4e). These results indicate that the double mutant was not able to prime the piglets for a secondary antibody response.

\section{DISCUSSION}

Bordetella represents a genus of pathogenic or opportunistic bacteria able to colonize the upper respiratory tract of a variety of warm-blooded animals. The most well studied species of this genus, $B$. pertussis, is a strictly human pathogen, whereas $B$. bronchiseptica colonizes a variety of different hosts and can cause respiratory disease in several different animals such as dogs and pigs. Most studies on Bordetella pathogenesis have been carried out using small animal models, such as rodents. Thus, little information is available on the role played by the toxins and adhesins produced by these bacteria in the pathogenesis of bordetellosis and colonization of Bordetella in their actual hosts. In this study, we analyzed the role played by Cya and Prn in the colonization of $B$. bronchiseptica in the respiratory tract of piglets and in the induction of anti-FHA antibody responses after i.n. inoculation.

Two mutant strains were isolated, one deficient for Cya and the other deficient for both Cya and Prn. We found that the single Cya-deficient mutant colonized the nasal cavity of the piglets to a lesser extent than the wild-type strain, and that the double mutant did not colonize the nasal cavity. According to previous studies, Cya targets neutrophils during infection [17]. Thus, it is possible the more rapid elimination of the Cya-deficient strain from the nasal cavity may have been due to the activity of the neutrophils at the early stages of infection.

We found that the wild-type strain persisted for six weeks in the nostrils; these results are consistent with those previous results [21]. The elimination of the bacteria from the nasal cavity correlated with the presence of anti-FHA IgM and IgA in the 
(a)

cya $B$ int

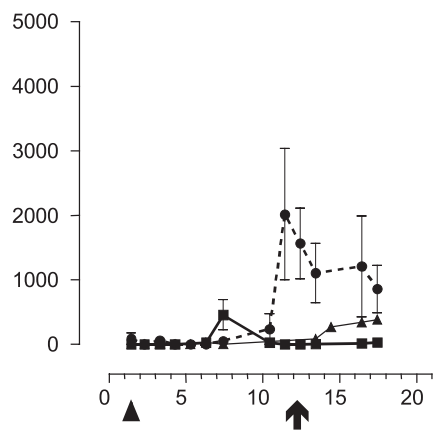

(b)

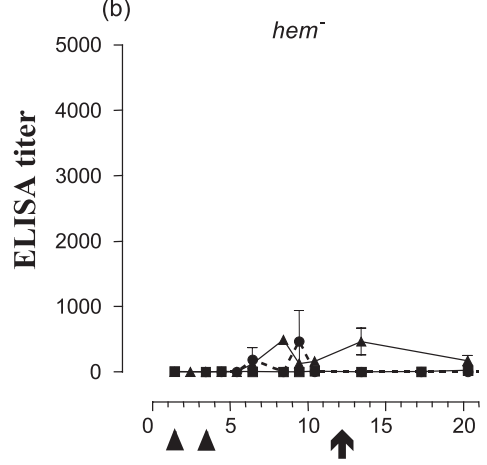

(c)

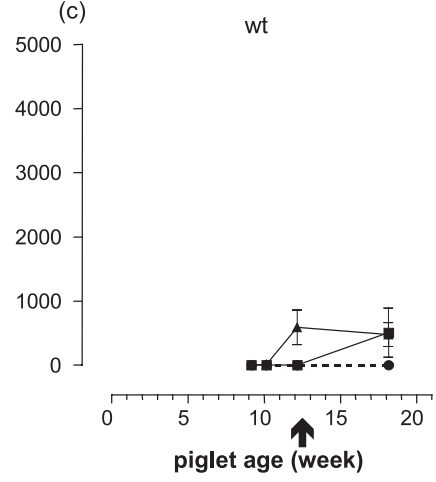

(d)

cya $B$ int

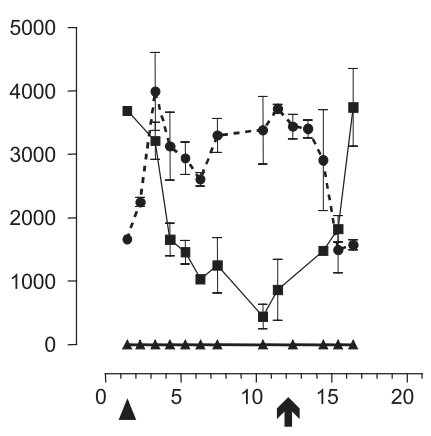

(e)
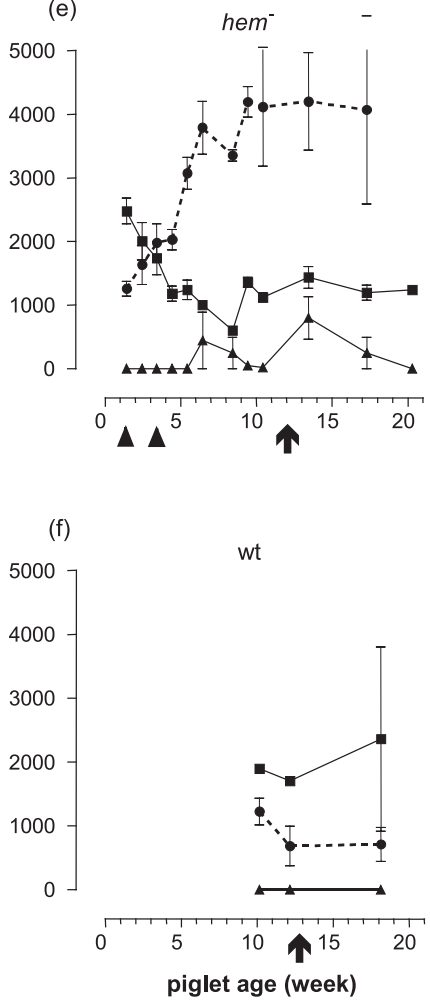

Figure 4. Anti-FHA antibody kinetics after boosting with i.n. B. bronchiseptica NL1013 inoculation. Piglets initially inoculated with $B$. bronchiseptica NL1013 cyaB int ( $n=3$, panels a and d) or NL1013 $h_{e m}^{-}(n=3$, panel $\mathrm{b}$ and $\mathrm{e})$ at one week of age, were then infected at 10 weeks of age with B. bronchiseptica NL1013. Unprimed piglets from another litter of the same age ( $n=2$, panels $\mathrm{c}$ and d) served as controls. Anti-FHA $\operatorname{IgA}(\boldsymbol{\Lambda}), \operatorname{IgG}(\boldsymbol{\square})$ and $\operatorname{IgM}(\bullet)$ levels were measured in the nasal secretions of each nostril (panels a, b and c) and in the sera (panels d, e and f) at the indicated time points after i.n. inoculation. The triangles indicate the time points of bacterial inoculation with the mutant strains (panels a, b, d, e) and $\uparrow$ indicates the time point of inoculation with B. bronchiseptica NL1013. The first sample was taken before inoculation to evaluate the pre-existing levels of anti-FHA antibodies. 
nasal secretions. These findings were consistent with those from a previous study, showing that IgM and IgA can prevent the attachment of bacterial cells [9].

In contrast to the wild-type strain, the Cya-deficient mutant colonized the nasal cavity of the neonate piglets for only two weeks. These results contrast with those obtained in rats and mice, where a Cya-deficient strain colonized the nasal cavity to the same extent as the wild-type strain [17]. This difference may be due to the fact that we used a relatively low ( 25 times less) volume of inoculum in our porcine study as compared to that used in the mouse studies [17]. However, B. bronchiseptica colonizes the nasal cavity of pigs to a much higher degree than it does the trachea and lung [1]. Thus, the use of low volume B. bronchiseptica inoculum in the piglet model, more closely mimics infection in the natural host. In addition, we found that when the wild type B. bronchiseptica strain was administered to 10 -week-old piglets, it was unable to colonize the nasal cavity. This fits with the view that, after 26 days of age, piglets become increasingly resistant due to the maturation of the epithelium [24].

The Cya- and Prn-deficient strain did not colonize the nasal cavity of neonate piglets and of the three strains was least able to adhere to epithelial cells in vitro, suggesting that the adhesin Prn plays a critical role in the colonization of the upper respiratory tract of piglets. However, since the number of HeLa cells found associated with this strain was only decreased by $25 \%$, relative to the wild type, other factors, such as FHA, are clearly also involved in mediating the attachment of B. bronchiseptica to cells.

Each litter used in this study contained maternally derived serum antibodies against the bacteria, a situation frequently observed in farm pigs [19]. Depending on their level in serum, these antibodies may interfere with nasal colonisation and may delay the immune response [21]. No anti-FHA antibodies were detected in the nasal secretions, indicating that no antibodies were trans- ferred from the serum and therefore that the maternal antibodies did not interfere with nasal colonization. These results are consistent with those obtained previously by Kono et al. [21]. In addition, the lack of an increase in anti-FHA IgG levels in piglets inoculated with the mutants may be associated with the influence of the maternally derived antibodies on the $\mathrm{IgG}$ response of the piglets. Kono et al. [21] found that maternally derived antibodies interfered with the IgG response in their study. This effect may be related to epitope masking of FHA by maternally derived anti-FHA IgG [38]; however, since there was an increase in anti-FHA IgM levels, this hypothesis seems unlikely. On the contrary, we showed that the B cells in piglets of the same age are able to switch from $\operatorname{IgM}$ to $\mathrm{IgG}$, indicating that they are mature [30]. Our results therefore indicate that the mutants lack immunogenicity (see below), a deficiency that may be due to a lower antigenic load and/or the CyA deficiency.

In neonate piglets infected with the B. bronchiseptica NL1013 wild type, antiFHA IgM and IgA production in the nasal cavity occurred much earlier than anti-FHA IgG production in the sera. There were three peaks in IgM and IgA levels in the nasal secretions, the last of these peaks occurs after bacteria were no longer detectable in the nasal secretions. This peak may be the result of a boost resulting from the presence of intracellular bacteria, since B. bronchiseptica can invade and persist in several eukaryotic cell lines, including dentritic cells [14, 15]. However, if the antibodies were trapped in immune complexes [19], it is also possible that we underestimated the amount of antibodies present at the time points between the peaks.

Our observations of both nasal and serum immune responses are consistent with the notion that the tonsils of piglets may be the inductive sites for the priming and dissemination of antigen-specific mucosal immune responses. In rodents, the associated lymphoid tissues consist of dense foci of lymphoid cells located in the nasal passage; 
whereas in swine [6] and humans, this tissue is comprised of the tonsils belonging to the Waldeyer ring [33]. During infection the wild type B. bronchiseptica strain adheres to the ciliated epithelial cells covering the dome of the follicles, and multiplies on the epithelium surface. This may result in direct stimulation of the M cells and the lymphoid cells of the follicles of palatine and/or pharyngeal tonsils and trigger the production of local IgM. Since the ability of the Cya and Prn double mutant to adhere to epithelial cells was impaired, this may have effected colonization of the epithelia in the vicinity of the tonsils resulting in this strain being unable to induce local antibody responses. In contrast, the ability of the CyA-deficient mutant to adhere to epithelial cells was similar to the wild type, and thus this strain may have gained access to the tonsil and been able to prime the lymphocytes in the follicles to mount an anamnestic response.

The fact that serum anti-FHA antibodies appeared during the later stages of infection with wild type $B$. bronchiseptica, at a time when the bacteria had been cleared from the nasal cavity, and earlier with the CyA-deficient strain, suggests that the IgG-producing precursor B cells may have disseminated from the inductive to the peripheral sites. Dissemination of lymphoid cells from the tonsils is supported by the finding that i.n. immunization in humans induces two types of antibody-secreting precursor cells in tonsil adenoids. Each of them utilizes a different set of homing receptors $[8,31,35]$. One type (IgG/IgA and $\left.\alpha_{4} \beta_{7}\right)$ matures in the tonsillar microenvironment, while the other type (IgG/IgA and L-selectin) migrates via circulation in the blood to other locations. Thus, the systemic response may be delayed as compared to the local response due to lower concentrations of immunoglobulinproducing cells. However, it is likely that the IgG precursor cells do not home in to the nasal mucosa to a great extent, since $\operatorname{IgA}$ is the predominant isotype in nasal secretions in young pigs $[6,31]$ and results mainly from local synthesis. In addition, other immunoglobulins may be present due to reverse passive transudation [32]. However, although we did not observe any clinical signs of pneumonia, we cannot exclude the possibility that the wild strain spread to the lung where it initiated a systemic IgG immune response.

It is of interest to note that the first rise in anti-FHA IgM and IgA levels in the nasal secretions occurred very soon after infection with the wild type B. bronchiseptica, and also that the CyA-deficient mutant primed the piglets to produce $\operatorname{IgM}$ in response to infection with the wild-type. In contrast, in the systemic response, IgM and IgA levels did not increase until 10 weeks after inoculation with the wild type strain and the CyA-deficient mutant primed the piglets to produce IgG in response to infection with the wild-type. These results suggest that only the wild-type strain induced the switch in production of $\operatorname{IgM}$ to IgA precursors in the tonsils and the expression of homing receptors for the nasal mucosa. In turn, this indicates that Cya may affect regulatory functions of the porcine immune system; it may affect the release of cytokines, such as TGF- $\beta$ or IL-12, or impair the release of innate effectors involved in the development of a systemic response [7].

\section{ACKNOWLEDGMENTS}

We thank P. Gueirard and N. Guiso (Institut Pasteur, Paris, France) for immunoblot analyses of the mutants, P. Bernardet, R. Delaunay, D. Musset and W. Piémont for technical help with the experimental infections, E. Veithen for FHA purification, F. Shali for technical assistance and M.H. Kobish for the gift of hyper immune serum. This work was supported by a CEE grant (FAIR, CT96-1339). L. Hibrand-Saint Oyant was supported by a postdoctoral fellowship from the CEE.

\section{REFERENCES}

[1] Ackermann M.R., Register K.B., GentryWeeks C., Gwaltney S.M., Magyar T., A porcine model for the evaluation of virulence of 
Bordetella bronchiseptica, J. Comp. Pathol. 116 (1997) 55-61.

[2] Antoine R., Locht C., Isolation and molecular characterization of a novel broad-host-range plasmid from Bordetella bronchiseptica with sequence similarities to plasmids from Grampositive organisms, Mol. Microbiol. 6 (1992) 1785-1799.

[3] Arico B., Rappuoli R., Bordetella parapertussis and Bordetella bronchiseptica contain transcriptionally silent pertussis toxin genes, J. Bacteriol. 169 (1987) 2847-2853.

[4] Arico B., Scarlato V., Monack D.M., Falkow S., Rappuoli R., Structural and genetic analysis of the bvg locus in Bordetella species, Mol. Microbiol. 5 (1991) 2481-2491.

[5] Bemis D.A., Wilson S.A., Influence of potential virulence determinants on Bordetella bronchiseptica-induced ciliostasis, Infect. Immun. 50 (1985) 35-42.

[6] Bourges D., Wang C.H., Chevaleyre C., Salmon H., T and IgA B lymphocytes of the pharyngeal and palatine tonsils: differential expression of adhesion molecules and chemokines, Scand. J. Immunol. 60 (2004) 338350.

[7] Boyaka P.N., Lillard J.W., Mcghee J., Interleukin 12 and innate molecules for enhanced mucosal immunity, Immunol. Res. 20 (1999) 207-217.

[8] Bradley P.A., Bourne F.J., Brown P.J., The respiratory tract immune system in the pig. I. Distribution of immunoglobulin-containing cells in the respiratory tract mucosa. II. Associated lymphoid tissues, Vet. Pathol. 13 (1976) 81-89.

[9] Cahill E.S., Ohagan D.T., Illum L., Barnard A., Mills K.H.G., Redhead K., Immuneresponses and protection against Bordetella pertussis infection after intranasal immunization of mice with filamentous hemagglutinin in solution or incorporated in biodegradable microparticles, Vaccine 13 (1995) 455-462.

[10] Chalfie M., Tu Y., Euskirchen G., Ward W.W., Prasher D.C., Green fluorescent protein as a marker for gene expression, Science 263 (1994) 802-805.

[11] Goodnow R.A., Shade F.J., Control of canine bordetellosis, Modern Vet. Pract. 61 (1980) 597-598.

[12] Gueirard P., Guiso N., Virulence of Bordetella bronchiseptica: role of denylalte cyclasehemolysin, Infect. Immun. 61 (1993) 40724078 .
[13] Gueirard P., Minoprio P., Guiso N., Intranasal inoculation of Bordetella Bronchiseptica in mice induces long-lasting antibody and T-cell mediated immune responses, Scand. J. Immunol. 43 (1996) 181-192.

[14] Guzman C.A., Rohde M., Bock M., Timmis K.N., Invasion and intracellular survival of Bordetella bronchiseptica in mouse dendritic cells, Infect. Immun. 62 (1994) 5528-5537.

[15] Guzman C.A., Rohde M., Timmis K.N., Mechanisms involved in uptake of Bordetella bronchiseptica by mouse dendritic cells, Infect. Immun. 62 (1994) 5538-5544.

[16] Harris D.L., Switzer W.P., Turbinate atrophy in young pigs exposed to Bordetella bronchiseptica, Pasteurella multocida and combined inoculum, Am. J. Vet. Res. 29 (1968) $777-785$.

[17] Harvill E.T., Cotter P.A., Yuk M.H., Miller J.F., Probing the function of Bordetella bronchiseptica adenylate cyclase toxin by manipulating host immunity, Infect. Immun. 67 (1999) 1493-1500.

[18] Jacob-Dubuisson F., Kehoe B., Willery E., Reveneau N., Locht C., Relman D.A., Molecular characterization of Bordetella bronchiseptica filamentous haemagglutinin and its secretion machinery, Microbiology 146 (2000) 1211-1221.

[19] Jenkins E.M., Anthony V., Vance R.T., Cleveland J., Gbadamosi S.G., Prevalence of Bordetella bronchiseptica infection in swine of southeastern Alabama, Am. J. Vet. Res. 38 (1977) 2071-2074.

[20] Kaeffer B., Bottreau E., Phan T.L., Olivier M., Salmon H., Histocompatible miniature, boar model: selection of transformed cell lines of $\mathrm{B}$ and $\mathrm{T}$ lineages producing retrovirus, Int. J. Cancer. 46 (1990) 481-488.

[21] Kono Y., Suzuki S., Mukai T., Okazaki K., Honda E., Yamashiro T., Detection of specific systemic and local IgG and IgA antibodies of pigs after infection with Bordetella bronchiseptica by ELISA, J. Vet. Med. Sci. 56 (1994) 249-253.

[22] Kovach M.E., Phillips R.W., Elzer P.H., Roop R.M., Peterson K.M., Pbbr1mcs: a broad-hostrange cloning vector, Biotechniques 16 (1994) 800-802.

[23] Kremer L., Baulard A., Estaquier J., Poulaingodefroy O., Locht C., Green fluorescent protein as a new expression marker in mycobacteria, Mol. Microbiol. 17 (1995) 913-922. 
[24] Larochelle R., Martineau-Doize B., Distribution of epithelia in the nasal cavity of piglets, Acta Anat. (Basel). 139 (1990) 214-219.

[25] Li Z.M., Hannah J.H., Stibitz S., Nguyen N.Y., Manclark C.R., Brennan M.J., Cloning and sequencing of the structural gene for the porin protein of Bordetella pertussis, Mol. Microbiol. 5 (1991) 1649-1656.

[26] Locht C., Antoine R., Jacob-Dubuisson F., Bordetella pertussis, molecular pathogenesis under multiple aspects, Curr. Opin. Microbiol. 4 (2001) 82-89.

[27] Menozzi F.D., Gantiez C., Locht C., Interaction of the Bordetella pertussis filamentous hemagglutinin with heparin, FEMS Microbiol. Lett. 62 (1991) 59-64.

[28] Menozzi F.D., Mutombo R., Renauld G., Gantiez C., Hannah J.H., Leininger E., Brennan M.J., Locht C., Heparin-inhibitable lectin activity of the filamentous hemagglutinin adhesin of Bordetella pertussis, Infect. Immun. 62 (1994) 769-778.

[29] Miller J.H., A short course in bacterial genetics, A laboratory manual and handbook for Escherichia coli and related bacteria, Cold Spring Harbor Laboratory Press, Plain View, New York, 1992.

[30] Morein B., Abusugra I., Blomqvist G., Immunity in neonates, Vet. Immunol. Immunopathol. 87 (2002) 207-213.

[31] Morgan K.L., Bourne F.J., Immunoglobulin content of the respiratory tract secretions of piglets from birth to 10 weeks old, Res. Vet. Sci. 31 (1981) 40-42.

[32] Morgan K.L., Hussein A.M., Newby T.J., Bourne F.J., Quantification and origin of the immunoglobulins in porcine respiratory tract secretions, Immunology 41 (1980) 729-736.

[33] Perry M., Whyte A., Immunology of the tonsils, Immunol. Today 19 (1998) 414-421.
[34] Quandt J., Hynes M.F., Versatile suicide vectors which allow direct selection for gene replacement in gram-negative bacteria, Gene 127 (1993) 15-21.

[35] Quiding-Jabrink M., Nordstrom I., Granstrom G., Kilander A., Jertborn M., Butcher E.C., Lazarovits A.I., Holmgren J., Czerkinsky C., Differential expression of tissue-specific adhesion molecules on human circulating antibody-forming cells after systemic, enteric, and nasal immunizations. A molecular basis for the compartmentalization of effector B cell responses, J. Clin. Invest. 99 (1997) 12811286.

[36] Sambrook J., Fritsch E.F., Maniatis T., Molecular cloning: a laboratory manual, 2nd ed., Cold Spring Harbor Laboratory, Press, Cold Spring Harbor, New York, 1989.

[37] Savelkoul P.H.M., Kremer B., Kusters J.G., Zeijst B.A.M., Van Der Gaastra W., Invasion of HeLa cells by Bordetella bronchiseptica, Microb. Pathog. 14 (1993) 161-168.

[38] Siegrist C.A., Mechanisms by which maternal antibodies influence infant vaccine responses: review of hypotheses and definition of main determinants, Vaccine 21 (2003) 3406-3412.

[39] Stainer D.W., Scholte M.J., A simple chemically defined medium for the production of phase I Bordetella pertussis, J. Gen. Microbiol. 63 (1970) 211-220.

[40] Van Den Akker W.M.R., Bordetella bronchiseptica has a BvgAS-controlled cytotoxic effect upon interaction with epithelial cells, FEMS Microbiol. Lett. 156 (1997) 239-244.

[41] Weiss A.A., Falkow S., Genetic analysis of phase change in Bordetella pertussis, Infect. Immun. 43 (1984) 263-269.

[42] Yuk M.H., Cotter P.A., Miller J.F., Genetic regulation of airway colonization by Bordetella species, Am. J. Respir. Crit. Care Med. 154 (1996) S150-S154. 